\title{
๖The Impact of GPS RO Data on the Prediction of Tropical Cyclogenesis Using a Nonlocal Observation Operator: An Initial Assessment
}

\author{
SHU-YA CHEN \\ GPS Science and Application Research Center, National Central University, Taoyuan, Taiwan \\ YING-HwA KUO \\ UCAR Community Programs, University Corporation for Atmospheric Research, Boulder, Colorado \\ CHING-YUANG HUANG \\ Department of Atmospheric Sciences, National Central University, Taoyuan, Taiwan
}

(Manuscript received 29 August 2019, in final form 3 April 2020)

\begin{abstract}
In this study, the impact of global positioning system (GPS) radio occultation (RO) data on the prediction of the genesis of 10 tropical cyclones over the western North Pacific Ocean is assessed. With the use of a nonlocal excess phase observation operator in cycling data assimilation, the probability of detection for tropical cyclogenesis is increased from $30 \%$ to $70 \%$ for the cases considered, all of which developed into typhoons. However, the probability of detection is only increased to $40 \%$ when a local observation operator is used, indicating that the observation operator can significantly influence the performance of RO data assimilation in capturing tropical cyclogenesis. A nonlocal excess phase operator, which considers the atmospheric horizontal gradients by integrating the refractivity along a ray path, gives superior performance over the local observation operator. Additional sensitivity experiments on 3 of the 10 typhoon cases show that the RO data in the vicinity of the incipient cyclones (within $500 \mathrm{~km}$ of the cyclone center) are most critical to successful cyclogenesis prediction. This reflects the fact that having good $\mathrm{RO}$ observations at the right time and place is critical for RO to have beneficial impacts on tropical cyclogenesis. Further analyses for Typhoon Nuri (2008) show that assimilation of RO data using the nonlocal operator leads to moistening of the lower and middle troposphere, organized convection, robust grid-scale vertical motions, and the development of midlevel relative vorticity, all of which are favorable for tropical cyclogenesis.
\end{abstract}

\section{Introduction}

An intense tropical cyclone (TC) is usually accompanied by strong winds and heavy precipitation, which can result in serious damage to agriculture, economy, and properties and loss of human life. An early warning of TC formation a few days before its actual genesis could allow more time for disaster preparedness. Numerical weather prediction (NWP) is the principal method to predict and detect tropical cyclogenesis. However, accurate prediction of cyclone formation-for example, several days in advance-is still a challenge. For example,

๑ Denotes content that is immediately available upon publication as open access.

Corresponding author: Ying-Hwa Kuo, kuo@ucar.edu
Tsai et al. (2011) studied the TC genesis over the western North Pacific (WNP) basin and found that the skill of a 0-96-h forecast for cyclogenesis is considerably higher than a longer-range forecast (102-384 h). Halperin et al. $(2013,2016)$ analyzed TC genesis forecasts in the North Atlantic Ocean basin and eastern North Pacific Ocean basin from several operational global models and showed that the success ratio (SR) of model-based TC genesis forecasts decreased with increasing forecast hour. Most of the models achieved a mean SR of less than 0.5 after a 72-h forecast, except for the European Centre for Medium-Range Weather Forecasts (ECMWF). Even though ECMWF had the best performance, its SR also decreased with increasing time.

Although the detecting skills of TC genesis in operational global models have been improved and become increasingly more reliable (Halperin et al. 2016), 
the model forecasts have been shown to be very sensitive to the initial conditions (e.g., Zhang and Sippel 2009; Doyle et al. 2012). Sippel and Zhang (2008) demonstrated that the presence of deep moisture and high convective available potential energy in the initial condition is very important for tropical cyclone formation. Doyle et al. (2012) used adjoint diagnostics to identify factors that are important to the prediction of tropical cyclogenesis, and they found that the accuracy of the forecast is most sensitive to the perturbations in moisture and temperature fields at the initial time. Furthermore, $\mathrm{Li}$ and $\mathrm{Pu}$ (2014) examined factors affecting Typhoon Nuri (2008) formation using the Weather Research and Forecasting (WRF) Model, and they also found the importance of initial conditions. In their study, forecasts initialized with the National Centers for Environmental Prediction (NCEP) Final (FNL) and ECMWF interim reanalysis (ERA-Interim) data gave very different results. Additional experiments showed that increasing the grid resolution to $4 \mathrm{~km}$ did not improve model skill for the prediction of Nuri's genesis.

A significant challenge for tropical cyclogenesis prediction is the lack of in situ observations over oceans. The global positioning system (GPS) radio occultation (RO) technique has many important attributes that can provide valuable observation over the ocean due to their global coverage and high vertical resolution. Since the GPS RO technique measures the time delay of signal phase passing through the atmosphere, it is minimally affected by clouds and precipitation and does not need calibration (Kursinski et al. 1997). The detail of GPS RO data processing procedures can be found in Kuo et al. (2004). GPS RO data have been widely used in NWP at the global operational weather centers, including ECMWF (Healy and Thépaut 2006), NCEP (Cucurull et al. 2007, 2013), Environment Canada (Aparicio and Deblonde 2008), Météo-France (Poli et al. 2008) and the Met Office (Rennie 2010). They have all shown positive impacts of RO data on global predictions, especially for the Southern Hemisphere where traditional observations are sparse. A study of forecast sensitivity to observation (FSO) by ECMWF (Cardinali 2009) indicated that GPS RO data contribute approximately $2 \%-3 \%$, in terms of data volume, of all the data assimilated, but GPS RO ranked 5 in terms of its impact on the reduction of model forecast errors (Cardinali 2013; Healy 2013). Based on the study from ECMWF, the RO data that contribute to the largest reduction on 24-h forecast errors are located over the upper troposphere and lower stratosphere (Healy 2013), which agrees with Rennie (2010).

Additional studies have demonstrated the impact of GPS RO data on climate monitoring, severe weather forecast, and verification (e.g., Huang et al. 2010; Anthes 2011;
Steiner et al. 2011; Cucurull et al. 2014). The positive influences on tropical cyclone prediction have been demonstrated in several other studies as well: Huang et al. (2005, 2010), Chen et al. (2009), Kueh et al. (2009), Liu et al. (2012), and Chen et al. (2015). To examine the impacts of GPS RO data on typhoon predictions over the western North Pacific, Chen et al. (2015) performed forecast experiments for 11 typhoon events during 2008-10. They showed that the assimilation of GPS RO refractivity improved the prediction of the western Pacific subtropical high and the associated circulation (i.e., steering flow), which subsequently reduced the forecast track error.

Most of the previous investigations of RO data impacts focus on cyclones that have already formed or developed. Very few studies discussed RO data impact on tropical cyclogenesis, except for Liu et al. (2012). They investigated the formation of Hurricane Ernesto (2006) over the Atlantic Ocean using an ensemble Kaman filter data assimilation system, and they found that the assimilation of GPS RO refractivity data increased the moisture in the vicinity of the tropical cyclone, increased the cyclonic circulation, and subsequently led to the genesis of the storm. Liu et al. (2012) have clearly indicated the importance of moisture in the lower tropical troposphere for accurate prediction of tropical cyclogenesis. However, the moisture in the lower tropical troposphere is highly variable, with significant horizontal and vertical structure that is difficult to capture. This presents a significant challenge for the GPS RO measurement technique, as well as the modeling of such measurement in a numerical model (i.e., observation operator). Currently, the local refractivity and local bending angle observation operators are often used in operational centers. The GPS RO local refractivity forward observation operator is simpler and less demanding in computation than the local bending angle observation operator. The retrieval of GPS RO refractivity, which is derived from bending angle under an assumption of local spherical symmetry, requires the use of climatological information (Kuo et al. 2004). In contrast, the bending angle retrieval, which is one step ahead of the refractivity in the retrieval process, does not need the climatological information (Cucurull et al. 2013; Huang et al. 2016). However, it still requires the spherical symmetry assumption. The local bending angle observation operator assimilates the GPS RO retrieved bending angle, assuming it is horizontally homogeneous, only a function of the impact parameter and neglecting the horizontal gradients in NWP models (Syndergaard et al. 2006). Neither of the local operators (refractivity and bending angle) consider the effect of atmospheric horizontal inhomogeneity. 
To take into account the effect of horizontal gradients, two-dimensional ray tracing operators have been developed recently (e.g., Healy et al. 2007; Wee et al. 2010), but it is more complicated and computationally demanding than with use of the local operators. Sokolovskiy et al. (2005a) proposed a nonlocal excess phase operator that integrates the GPS refractivity along a ray path in order to reduce the representativeness errors over the region with large horizontal refractivity gradients (due to moisture variations). Chen et al. $(2009,2011)$ have implemented the Sokolovskiy et al. nonlocal excess phase operator into the WRF data assimilation (DA) system. The performance of GPS RO assimilation can be sensitive to the observation operator used in the assimilation, particularly for the tropical cyclone prediction, as the observation operator reflects the accuracy of modeling the observables. In this study, we examine the impact of the RO operators on cyclogenesis prediction and compare the performance of the observation operators for the assimilation of GPS RO data. A comprehensive evaluation of all possible observation operators for NWP application is beyond the scope of this paper. In this paper, we only compare the local refractivity operator and the nonlocal excess observation operator for their impact on tropical cyclogenesis. To ensure that the results are statistically meaningful, assimilation and forecast experiments are performed on 10 tropical cyclogenesis cases over the western North Pacific.

The model and GPS RO operators are discussed in section 2. The data assimilation experiments and the forecast results are described in section 3 . The sensitivity of cyclogenesis prediction to the GPS RO soundings in the vicinity of an incipient cyclone is assessed as well. Section 4 presents an analysis of the impact of RO data on tropical cyclogenesis, using Typhoon Nuri (2008) as an example. Conclusions are given in section 5 .

\section{Forecast model and GPS RO operators}

\section{a. Numerical model configuration}

The numerical model used in this study is the Advanced Research version of WRF (WRF-ARW, hereinafter the WRF Model; Skamarock et al. 2008). The WRF Model is fully compressible and nonhydrostatic, which is suitable for diverse applications across a wide range of spatial scales. In addition, a WRF data assimilation system (WRFDA; Barker et al. 2012) has been developed for the WRF Model supporting variational (three- and fourdimensional frameworks, i.e., 3DVAR and 4DVAR) (Barker et al. 2004; Huang et al. 2009), ensemble Kalman filter (Anderson 2010), and hybrid approaches (Wang et al. 2008a,b). In this study, we use WRF 3DVAR, as it

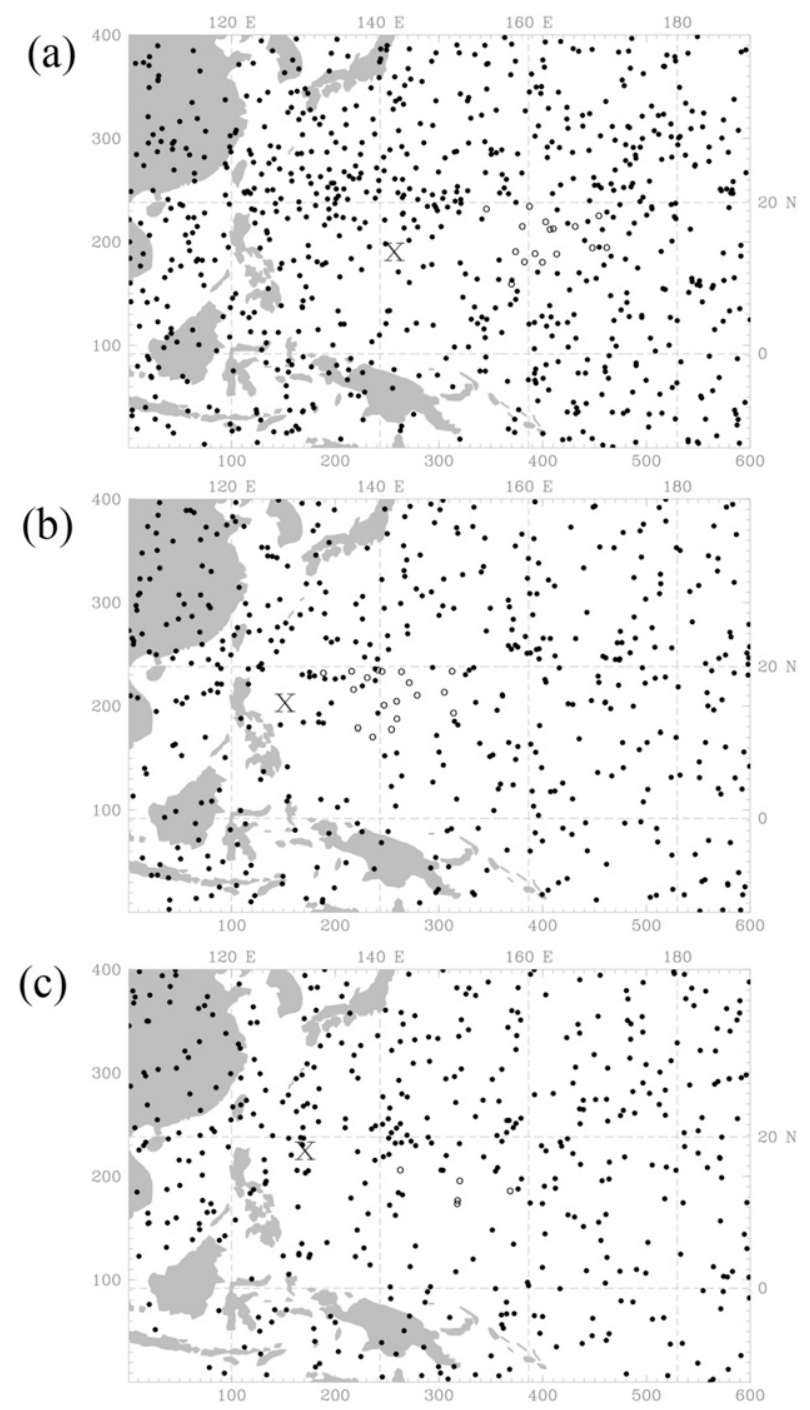

FIG. 1. Locations of GPS RO soundings within 3 days of data assimilation for Typhoons (a) Nuri (2008), (b) Sinlaku (2008), and (c) Fanapi (2010). The X indicates the location of JTWC's tropical depression (i.e., observed cyclogenesis), and the open circles are the GPS RO data near the region of incipient cyclone.

serves the purpose of our study with modest computational cost. The nonlocal excess phase operator, which involves integrations along ray paths, will be described in section 2b. Both the WRF and WRFDA used in this study are version 3.3.1. A detailed description of the WRF Model and WRFDA can be found online (http://wrf-model.org).

In this study, we use a single domain with horizontal resolution of $15 \mathrm{~km}$ on $600 \times 400$ grid cells (Fig. 1), and the domain coverage is similar to the outermost domain of 36-km resolution in $\mathrm{Li}$ and $\mathrm{Pu}$ (2014). $\mathrm{Li}$ and $\mathrm{Pu}$ (2014) showed that a higher-resolution grid spacing $(4 \mathrm{~km})$ does not help the simulation of cyclogenesis. 
The WRF Model has 45 layers in the vertical with the model top at $30 \mathrm{hPa}$. The NCEP FNL analysis $\left(0.5^{\circ} \times\right.$ $0.5^{\circ}$ resolution) is used to provide the initial and lateral boundary conditions. The model physics options include Goddard cloud microphysics scheme (Lin et al. 1983; Rutledge and Hobbs 1984), the Rapid Radiative Transfer Model (RRTM; Mlawer et al. 1997), the Goddard shortwave radiation scheme (Chou and Suarez 1994), the unified Noah land surface model (Chen and Dudhia 2001), the Yonsei University (YSU) planetary boundary layer parameterization scheme (Hong et al. 2006), and the Kain-Fritsch (Kain and Fritsch 1990, 1993) cumulus parameterization.

\section{b. GPS RO forward operators and the assimilation procedure}

Several data products are available from the GPS RO data processing chain, including bending angle, refractivity, derived temperature, pressure, and moisture (Kuo et al. 2004). Bending angle and refractivity are most widely used in both research and operational assimilation of RO data (e.g., Cucurull et al. 2007, 2013; Aparicio and Deblonde 2008; Poli et al. 2008; Rennie 2010; Chen et al. 2014; Yang et al. 2014; Huang et al. 2016). To assimilate either bending angle or refractivity observations in a data assimilation system, a corresponding forward operator is needed. The local refractivity operator is relatively simple. The atmospheric refractivity $N$ is related to several meteorological variables (Lewis 2008), such as

$$
N=77.6 \frac{P}{T}+3.73 \times 10^{5} \frac{P q}{T^{2}(0.622+0.378 q)},
$$

where $P$ is the pressure of the atmosphere $(\mathrm{hPa}), T$ is temperature (K), and $q$ is the specific humidity $\left(\mathrm{kg} \mathrm{kg}^{-1}\right)$. Recently, a revised three-term expression for refractivity was proposed and has been applied in operational centers, for example, Environment Canada and Météo-France (Lewis 2008).

The GPS RO refractivity is derived based on the assumption of local symmetry, which is not valid over areas with significant horizontal gradients. The nonlocal excess phase operator (Sokolovskiy et al. 2005a) takes into consideration the atmospheric horizontal refractivity variations by integrating the GPS RO refractivity using a constant step of $5 \mathrm{~km}$ along a straight line representing the ray path (Sokolovskiy et al. 2005b; Chen et al. 2009). The total integration length is typically about $1000 \mathrm{~km}$, or until the integration hits the model top and must be stopped. The integrated refractivity, that is, a new observable called pseudoexcess phase, or $S$, is defined as [Fig. 1 in Sokolovskiy et al. (2005a)]

$$
S=\int N d l
$$

where $l$ is the ray path. The assimilation of GPS RO data using the local refractivity operator and the nonlocal excess phase operator do not require the information above the model top.

Most operational weather prediction centers make use of local bending angle operators (Cucurull et al. 2013). For the bending angle operator, an estimate of the atmospheric property above the model is needed and is obtained through extrapolation (Healy and Thépaut 2006). This can potentially introduce some uncertainties, as the model top of $30 \mathrm{hPa}$ in this study is much lower than that of typical operational global models. In addition, both the nonlocal and local operators use the retrieved GPS RO refractivity as inputs; they are more comparable with each other than with the bending angle operator, which uses the GPS RO bending angle as input. Therefore, in this paper we focus on the comparison between local refractivity operator (LOC) and nonlocal excess phase operator (EPH) for the GPS RO data assimilation. Chen et al. (2009) had implemented the nonlocal operator into the WRFDA, which calculates GPS excess phase on the mean altitude of each model layer. In this study, we revise the process and calculate the nonlocal excess phase on the observed height (i.e., the mean sea level altitude) to make it consistent with the assimilation of local refractivity.

We now describe the assimilation procedures, including forward operator, observational error, and data quality control (QC). Both of the observables, that is, local refractivity and nonlocal excess phase, are calculated at the observation heights of about $200-\mathrm{m}$ vertical resolution. The 200-m resolution of the GPS RO data is comparable to that of the World Meteorologi cal Organization (WMO) Binary Universal Form for Representation of Meteorological Data (BUFR) format, which is used at operational centers. For the observation error, the error of refractivity depends on both altitude and latitude, which is the default configuration in WRFDA. For a sounding located at the equator, the percentage error for the local refractivity is $2.5 \%$ from the surface to the height of $2.5 \mathrm{~km}$ and then linearly decreases to $1.3 \%$ at $5.5 \mathrm{~km}$. It continues to decrease to $0.3 \%$ at $12 \mathrm{~km}$. For a sounding located at the pole, it is $1.5 \%$ near the surface and linearly decreases to $0.3 \%$ at $12 \mathrm{~km}$. All the observation errors above $12 \mathrm{~km}$ have the same constant value of $0.3 \%$. Then, the observational error of a RO sounding located between the equator and pole is obtained from linear interpolation (Fig. 2). For the nonlocal excess phase, the statistical observation error provided in Chen et al. (2009, their Fig. 1b) is used, 


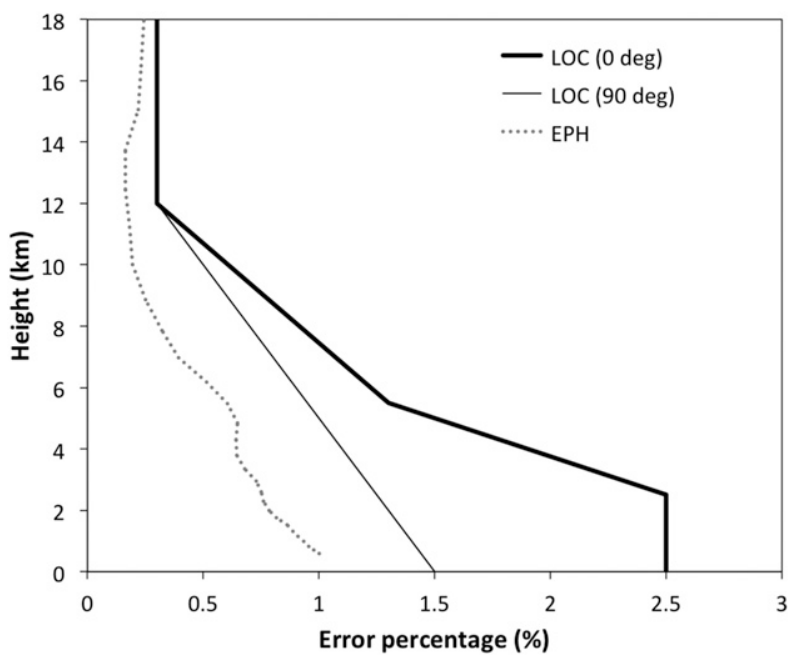

FIG. 2. The statistical observation errors for the local (LOC) and nonlocal (EPH) operators. The observational error in the assimilation system is related to both the altitude and latitude for the local operator but is only related to the altitude for the nonlocal operator.

which depends on altitude only. According to the error estimation in Chen et al. (2011), the observation error for the excess phase is not sensitive to latitude in the summer season and thus we assimilate the RO data for typhoon cases without latitudinal variation for EPH. The error in EPH is smaller in magnitude than that in LOC (Fig. 2). Different assimilation variables should have their own observation errors; therefore, we use their corresponding observational errors in the data assimilation. For the quality control, the LOC and EPH use the same gross check criterion; that is, the innovation has to be smaller than a predefined ratio or the data will be rejected.

\section{c. Assimilation of a single $R O$ profile}

To understand the behaviors of the two GPS RO operators, one RO sounding is chosen for the assimilations with the EPH and LOC, respectively. The sounding was obtained at 2245:08 UTC 13 August 2008 and located at $7.32^{\circ} \mathrm{N}, 158.26^{\circ} \mathrm{E}$. Figure 3 shows the analysis increments for LOC and EPH, respectively. Assimilation with the nonlocal operator shows elliptical increments of the water vapor mixing ratio with a northeast-southwest orientation along the ray path (Fig. 3b), whereas the LOC shows more circular increments (Fig. 3a). On the cross section along the ray path indicated in Fig. 3b, the patterns and magnitudes of the temperature and moisture increments are similar and comparable for both operators (Figs. 3c-f). Both show a negative moisture increment below $6 \mathrm{~km}$ and a positive moisture increment above $6 \mathrm{~km}$. It is interesting to note that EPH gives a larger moisture decrease below $6 \mathrm{~km}$ and smaller moisture increase above $6 \mathrm{~km}$, compared with LOC. For the temperature, both have positive increments below $12 \mathrm{~km}$ and negative increments above $12 \mathrm{~km}$. The lowest height of this GPS RO sounding is $1935 \mathrm{~m}$, therefore only small increments exist below $2 \mathrm{~km}$. A comparison between moisture and temperature analysis increments clearly shows that assimilation of GPS RO data gives much larger change in moisture than temperature. This is because moisture is responsible for most of the variation in refractivity in the tropical lower troposphere. Also, the magnitude and distribution of analysis increments vary depending on the observation operators used in the assimilation. Both operators take into consideration the drifting of the perigee points with height of the RO sounding for the data assimilation.

To assess the performance of these two operators, a collocated radiosonde at $6.97^{\circ} \mathrm{N}, 158.22^{\circ} \mathrm{E}$, which was observed about $1.25 \mathrm{~h}$ later than the GPS RO event, is used as independent verification. Figure 4 shows the difference between the radiosonde and the first guess (FG), along with the analyses of EPH and LOC. For moisture, the EPH shows an obviously smaller difference than LOC (Fig. 4a). On the other hand, the patterns in temperature are very similar below $10 \mathrm{~km}$ (Fig. 4b) since the increments are small (Figs. 3e,f). Near the tropopause, the differences with radiosonde are reduced in the $\mathrm{RO}$ assimilation with either operator (LOC or EPH) when compared with that of the FG. In general, EPH has a smaller moisture difference with the radiosonde in the lower troposphere and a smaller temperature difference in the upper troposphere. Two additional radiosonde soundings collocated with $\mathrm{RO}$ were verified as well, and EPH consistently showed better fit than LOC (figures not shown). In summary, the results from the single observation assimilation indicate that EPH may improve both temperature and moisture analyses throughout the troposphere.

\section{Experiments and statistical results}

\section{a. Typhoon cases and experimental design}

To assess the GPS RO data impact on the genesis of WNP tropical cyclones, we selected 10 typhoon cases during 2008-10. The impact of GPS RO data on nondeveloping cases (and thus, the issue of false alarms) are not studied in this paper. The maximum intensities of all the 10 typhoon cases were stronger than category 1 , that is, a maximum wind speed exceeding $32.7 \mathrm{~m} \mathrm{~s}^{-1}$. They are Kalmaegi (2008), Fungwong (2008), Nuri (2008), Sinlaku (2008), Hagupit (2008), Jangmi (2008), Morakot (2009), Parma (2009), Fanapi (2010), and Megi (2010), where Typhoons Nuri, Sinlaku, and Hagupit developed within The Observing System Research and Predictability 

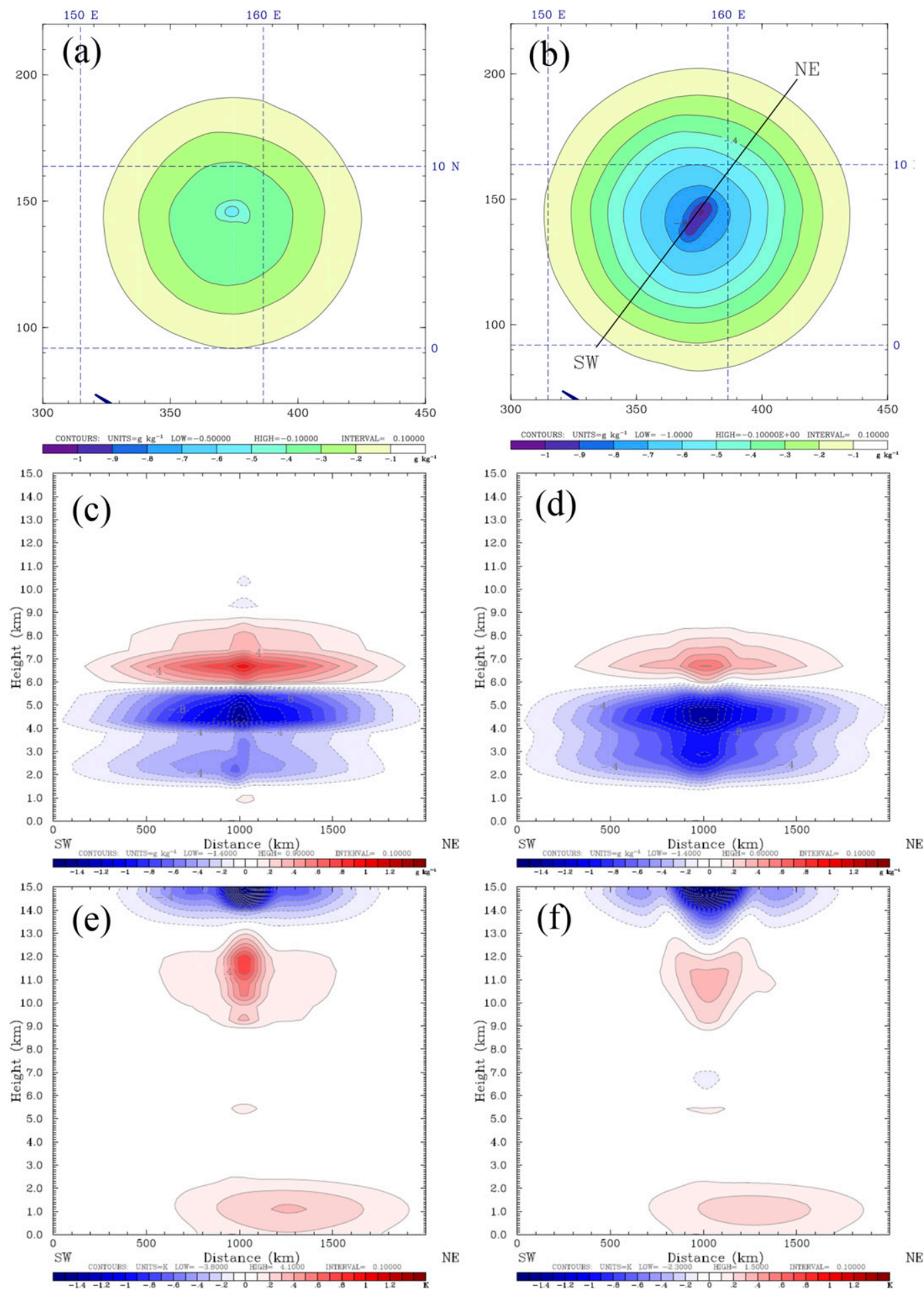

FIG. 3. Increments of water vapor mixing ratio at $700 \mathrm{hPa}$ for (a) LOC and (b) EPH. Also shown are increments of (b),(c) water vapor mixing ratio and (e),(f) temperature on the vertical cross section along the line in (b) for (left) LOC and (right) EPH. 

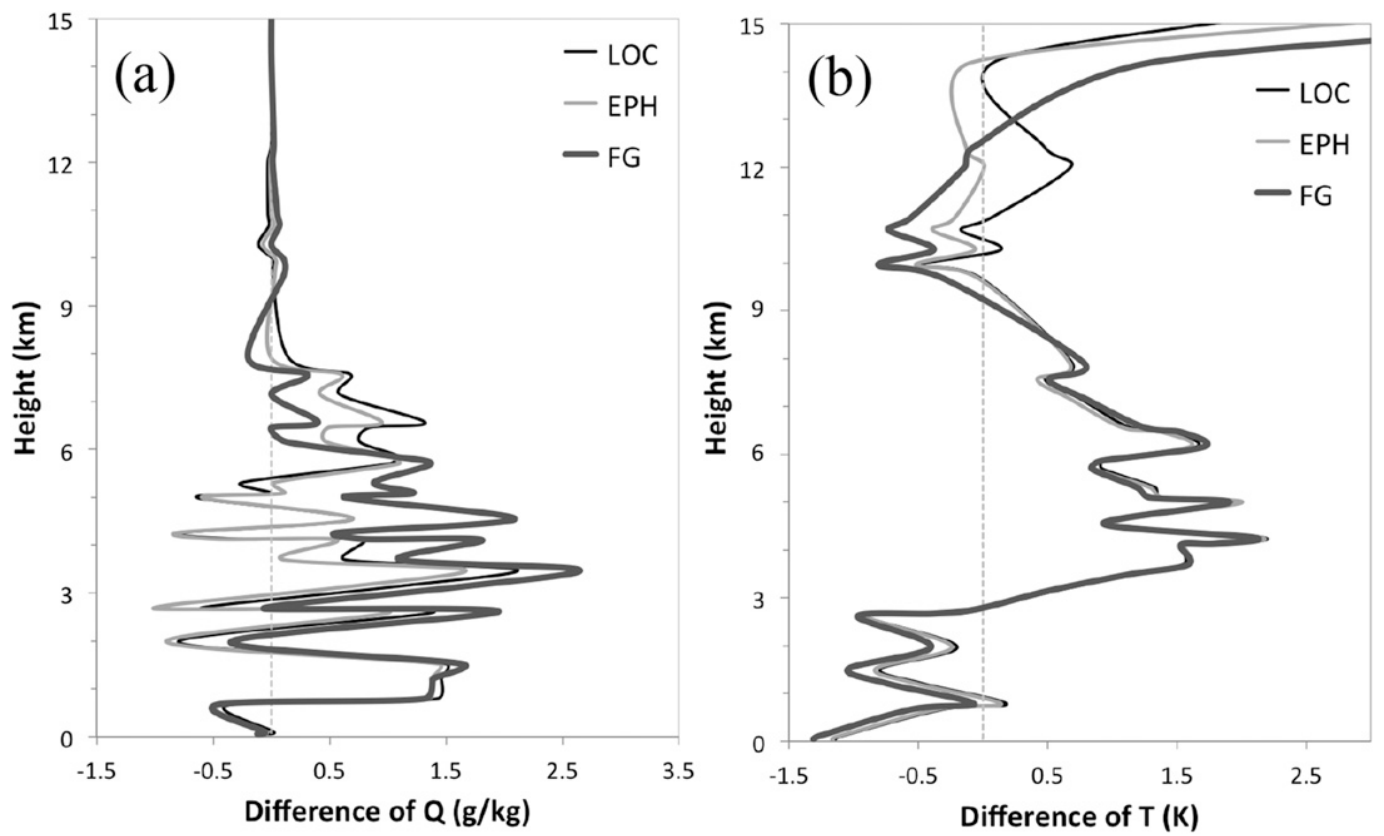

FIG. 4. The verification against radiosonde in (a) water vapor mixing ratio $\left(\mathrm{g} \mathrm{kg}^{-1}\right)$ and (b) temperature (K) for the first guess from NCEP (thick dark-gray line), and the analyses from LOC (thin black line) and EPH (thin light-gray line).

Experiment (THORPEX) Pacific Asian Regional Campaign (T-PARC) period in 2008. In this study, we assimilate the conventional data (e.g., radiosonde soundings, surface observations, and aircraft data, etc.) and a few satellite data retrievals (e.g., Quick Scatterometer surface winds and atmospheric motion vectors). The satellite radiance data are not assimilated. GPS RO data are obtained from the Constellation Observing System for Meteorology, Ionosphere and Climate (COSMIC) Data Analysis and Archive Center (CDAAC), which include the Challenging Minisatellite Payload (CHAMP), Satellite for Scientific Applications-C (SAC-C), Gravity Recovery and Climate Experiment (GRACE), Meteorological Operational Satellite- $A$ (MetOp-A), X-band TerraSAR satellite (TerraSAR-X), and the Formosa Satellite Mission 3 (FORMOSAT-3)/COSMIC (COSMIC 2013).

To define the time of cyclogenesis, we follow the Joint Typhoon Warning Center (JTWC) definition as the time of tropical depression (TD) formation. The numerical experiments for each case start at five days $(120 \mathrm{~h})$ prior to the genesis of TD. For each of the 10 typhoon cases we perform three experiments. The first two examine the impact of GPS RO observational operators, that is, LOC and EPH, by assimilating GPS RO data together with all the other observations as mentioned above. The third experiment is a data-denial experiment by assimilating the same data [i.e., Global Telecommunication System (GTS)] as the first two experiments, without the
RO data. The specific times of the cyclogenesis in JTWC and that in the corresponding WRF forecasts for the 10 typhoon cases are listed in Table 1 . There are more than $600 \mathrm{RO}$ soundings on average within the model domain for the typhoon cases before 2010, and the data volume is decreased afterward (Table 2). Four cycling assimilation cycles per day, each with a time window of $6 \mathrm{~h}$, are performed for each experiment. After 3 days of cycling data assimilation, a WRF Model free forecast of more than 4 days is conducted (Fig. 5).

A detection of cyclogenesis for each experiment was processed by a typhoon tracking algorithm in version 4

TABLE 1. Cyclogenesis for the 10 typhoon cases. A genesis time relative to the JTWC's TD genesis is indicated for each experiment (GTS, LOC, and EPH). The $\mathrm{x}$ indicates no cyclone formation.

\begin{tabular}{llccr}
\hline \multicolumn{1}{c}{ Typhoon } & \multicolumn{1}{c}{ JTWC TD genesis } & GTS & LOC & EPH \\
\hline Kalmaegi (2008) & 0000 UTC 14 Jul 2008 & $-48 \mathrm{~h}$ & $-42 \mathrm{~h}$ & $-42 \mathrm{~h}$ \\
Fungwong (2008) & 0600 UTC 24 Jul 2008 & $-36 \mathrm{~h}$ & $-36 \mathrm{~h}$ & $-42 \mathrm{~h}$ \\
Nuri (2008) & 1800 UTC 16 Aug 2008 & $\mathrm{x}$ & $30 \mathrm{~h}$ & $0 \mathrm{~h}$ \\
Sinlaku (2008) & 0000 UTC 8 Sep 2008 & $\mathrm{x}$ & $\mathrm{x}$ & $6 \mathrm{~h}$ \\
Hagupit (2008) & 1800 UTC 18 Sep 2008 & $24 \mathrm{~h}$ & $0 \mathrm{~h}$ & $-12 \mathrm{~h}$ \\
Jangmi (2008) & 1200 UTC 23 Sep 2008 & $6 \mathrm{~h}$ & $6 \mathrm{~h}$ & $-12 \mathrm{~h}$ \\
Morakot (2009) & 1800 UTC 3 Aug 2009 & $-48 \mathrm{~h}$ & $-24 \mathrm{~h}$ & $-6 \mathrm{~h}$ \\
Parma (2009) & 1200 UTC 27 Sep 2009 & $-6 \mathrm{~h}$ & $6 \mathrm{~h}$ & $-12 \mathrm{~h}$ \\
Fanapi (2010) & 1200 UTC 14 Sep 2010 & $\mathrm{x}$ & $\mathrm{x}$ & $36 \mathrm{~h}$ \\
Megi (2010) & 1800 UTC 12 Oct 2010 & $36 \mathrm{~h}$ & $60 \mathrm{~h}$ & $24 \mathrm{~h}$ \\
Predictability & & $30 \%$ & $40 \%$ & $70 \%$ \\
\hline
\end{tabular}


TABLE 2. The number of GPS RO soundings in the model domain at each assimilating time window.

\begin{tabular}{|c|c|c|c|c|c|c|c|c|c|c|c|c|c|c|}
\hline Typhoon case & DA1 & DA2 & DA3 & DA4 & DA5 & DA6 & DA7 & DA8 & DA9 & DA10 & DA11 & DA12 & DA13 & Total \\
\hline Kalmaegi (2008) & 45 & 6 & 35 & 38 & 57 & 27 & 39 & 34 & 52 & 37 & 36 & 34 & 60 & 500 \\
\hline Fungwong (2008) & 21 & 5 & 35 & 54 & 44 & 72 & 49 & 52 & 32 & 60 & 64 & 47 & 24 & 606 \\
\hline Nuri (2008) & 60 & 49 & 53 & 63 & 59 & 40 & 44 & 78 & 58 & 56 & 53 & 65 & 52 & 730 \\
\hline Sinlaku (2008) & 21 & 33 & 40 & 31 & 19 & 42 & 43 & 33 & 34 & 41 & 50 & 42 & 45 & 474 \\
\hline Hagupit (2008) & 49 & 40 & 52 & 58 & 51 & 41 & 61 & 75 & 49 & 66 & 53 & 65 & 36 & 696 \\
\hline Jangmi (2008) & 64 & 35 & 51 & 57 & 62 & 53 & 39 & 39 & 58 & 40 & 50 & 37 & 69 & 654 \\
\hline Morakot (2009) & 61 & 54 & 35 & 54 & 58 & 57 & 37 & 63 & 48 & 42 & 38 & 62 & 52 & 661 \\
\hline Parma (2009) & 26 & 36 & 51 & 46 & 58 & 42 & 54 & 57 & 53 & 37 & 58 & 44 & 62 & 624 \\
\hline Fanapi (2010) & 34 & 23 & 34 & 46 & 38 & 18 & 26 & 26 & 37 & 25 & 47 & 48 & 41 & 443 \\
\hline Megi (2010) & 30 & 38 & 31 & 33 & 24 & 43 & 25 & 28 & 15 & 42 & 35 & 26 & 23 & 393 \\
\hline
\end{tabular}

of the Read/Interpolate/Plot (RIP4) software package. Several criteria in the RIP4 must be met before a cyclone formation is declared. The criteria include a tropical cyclone formed over water; a closed low with a sea level pressure lower than $1004 \mathrm{hPa}$; the detection of a storm center according to temperature, wind speed, vorticity, etc.; surface temperature higher than $280 \mathrm{~K}$; 700 -hPa temperature higher than $1{ }^{\circ} \mathrm{C}$; and maximum vorticity at $700 \mathrm{hPa}$ greater than $1 \times 10^{-4} \mathrm{~s}^{-1}$ in the defined center. Since the loci of vortex centers based on above criteria may differ slightly, the final vortex position is defined by weighting the loci based on sea level pressure, 700-hPa vorticity, and 10-m wind speed. More detail of the typhoon tracking criteria can be found in the RIP4 package (http://www2.mmm.ucar.edu/wrf/ users/docs/ripug.htm). After a simulated cyclone meets all of these criteria, the associated maximum 10-m wind speed has to be higher than $25 \mathrm{kts}\left(12.87 \mathrm{~m} \mathrm{~s}^{-1}\right)$ to satisfy a successful simulation of cyclone formation.

\section{b. Statistical results and the importance of observation near the incipient cyclone}

Table 1 lists the time of vortex formation for each experiment, relative to the JTWC's genesis time. A positive hour indicates a delayed TD genesis in the simulation, a negative hour indicates an early prediction of the cyclone formation, and a zero hour indicates a perfect match of genesis time as reported in JTWC. The TD genesis can be simulated in most cases with or without the assimilation of the RO data; however, the timing can be significantly off (Table 1). Note that the cyclogenesis is captured by EPH for all 10 typhoon cases, but 3 typhoon cases [Nuri (2008), Sinlaku (2008), and Fanapi (2010)] are failed by GTS and 2 cases are failed by LOC. Following Halperin et al. (2013), we define a hit, a successful genesis prediction, if the model cyclone forms within a \pm 24 -h time window of the actual TD genesis as defined by the JTWC. Based on this definition, the probability of detection (POD) is $70 \%$ for EPH, $40 \%$ for LOC, and $30 \%$ for GTS (Table 1 ). Both experiments with GPS RO data assimilation (LOC and EPH) have higher PODs than that without RO data assimilation (GTS). It is worth noting that EPH has more than doubled the POD in cyclogenesis than GTS. It also shows the observation operator can have a significant influence on the prediction of tropical cyclogenesis. The use of the nonlocal operator EPH results in the highest POD. In addition, EPH did not miss any genesis captured by LOC or GTS for the 10 typhoon cases.

Observation in the vicinity of the incipient storm may play an important role in cyclogenesis prediction. We investigate the influences of GPS RO data in the vicinity of the incipient cyclone for Typhoons Nuri (2008),

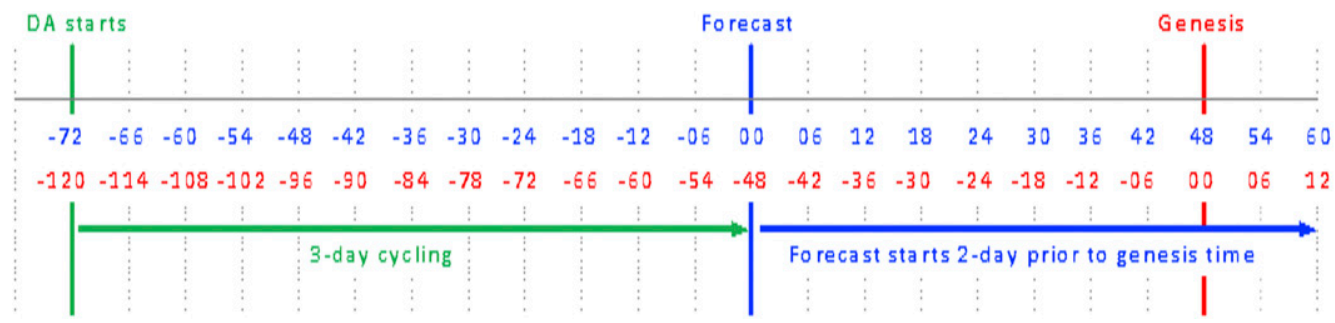

FIG. 5. Experimental design. Each simulation was carried out by 3-day cycling data assimilation and then forecast for more than $48 \mathrm{~h}$, that is, the time of a tropical depression genesis identified in JTWC (red digits). The forecast hour is indicated by blue digits. 
Sinlaku (2008), and Fanapi (2010). For these three cases, EPH has successfully captured the genesis, but GTS failed. To understand the impact of RO data near the storm, we define a box with the size of $10^{\circ} \times 10^{\circ}$ centered on the 500-hPa vortex. There are 16, 18, and 5 GPS RO soundings in the vicinity of the incipient vortices during the 3-day DA period for Typhoons Nuri, Sinlaku, and Fanapi, respectively. The loci of GPS RO data for the three typhoon cases are shown in Fig. 1. A cross sign indicates the TD genesis position from JTWC, and the RO soundings near the incipient cyclone, within the box as defined earlier, during the assimilation period are marked as open circles. We found that by removing the GPS RO data in the vicinity of the incipient storm from the assimilation, the EPH fail to predict the genesis for all the three cases. This indicates that the GPS RO data near the incipient storm are quite important for the prediction of cyclogenesis. To gain insights into the impact of the GPS RO data on the prediction of tropical cyclogenesis, a detailed analysis of the simulations is conducted for Typhoon Nuri (2008) in the next section.

\section{Typhoon Nuri (2008)}

\section{a. A brief overview of Typhoon Nuri (2008)}

Typhoon Nuri (2008) originated from an easterly wave over the WNP and the incipient disturbance was tracked by JTWC beginning at 0000 UTC 16 August 2008. After $18 \mathrm{~h}$ (i.e., 1800 UTC 16 August), the system reached the intensity of a tropical depression with a minimum sea level pressure of $1004 \mathrm{hPa}$ and a maximum surface wind speed of $12.8 \mathrm{~m} \mathrm{~s}^{-1}$. Then, it moved westward and developed into a tropical storm by 1200 UTC 17 August. Several studies have discussed the genesis mechanism of Typhoon Nuri (2008), for example, Raymond and López Carrillo (2011), Lussier et al. (2014), and $\mathrm{Li}$ and $\mathrm{Pu}$ (2014). Li and $\mathrm{Pu}$ (2014) found that different model initial conditions lead to different performance on the prediction of Nuri's genesis, reflecting the importance of the initial condition. They also found that both sufficiently warm SSTs and weak vertical wind shear are necessary, but not sufficient, for the genesis of Nuri. Their results show that mid- to upper-level moisture is an important factor that is favorable for Nuri's genesis.

\section{b. Results and analyses}

The geographical distributions of 730 GPS RO soundings during the 3-day assimilation for Nuri are shown in Fig. 1a. Figure 6 shows the vertical distribution of GPS RO data before and after QC that are actually assimilated into the WRFDA. The assimilated RO data

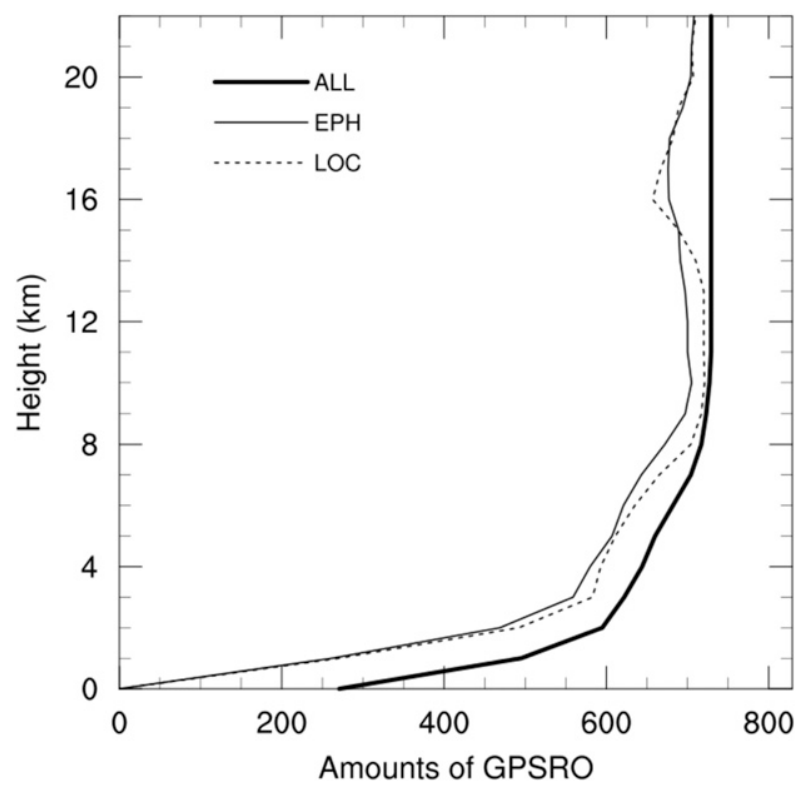

FIG. 6. Vertical variations of RO data amounts for Typhoon Nuri (2008). The RO data available before QC process during the DA period are indicated by ALL (thick line). After QC, LOC (dashed line) and EPH (thin line) indicate the actual use in WRFDA for each operator.

amounts change only slightly above $5 \mathrm{~km}$ for both operators, while they decrease rapidly in the lower troposphere. Because a similar QC procedure is employed for both operators, their usage in data assimilation is comparable. Thus, the differences in forecasts of Nuri for EPH and LOC should not be attributed to the small differences in $\mathrm{RO}$ data counts.

Figure 7 compares the ECMWF analysis and WRF 48-h prediction, from different experiments, of the sea level pressure and 10-m wind speed at 1800 UTC 16 August 2008. The horizontal resolution of the ECMWF analysis is about $25 \mathrm{~km}$. The 48 -h WRF forecasts (Fig. 7b), initialized with the ECMWF analysis, gives weaker winds in the vortex region than the verifying ECMWF analysis at that time (Fig. 7a). None of the 48-h WRF forecast from ECMWF analysis, GTS, or LOC (Figs. 7b-d) produce an organized vortex with closed isobars at the sea level. And, the simulated wind in the vicinity of the incipient cyclone is weak. A lot of additional observations and satellite radiances have been assimilated into the ECMWF or NCEP analyses that are used as the WRF initial conditions, but they still fail to produce the genesis of Typhoon Nuri (Figs. 7b-d). On the other hand, EPH predicts a well-organized vortex with stronger winds associated with the incipient cyclone, which has a sea level pressure of $1004 \mathrm{hPa}$ (Fig. 7e). The wind field structures are similar for LOC and EPH (Figs. 7d,e), but the former in general is 

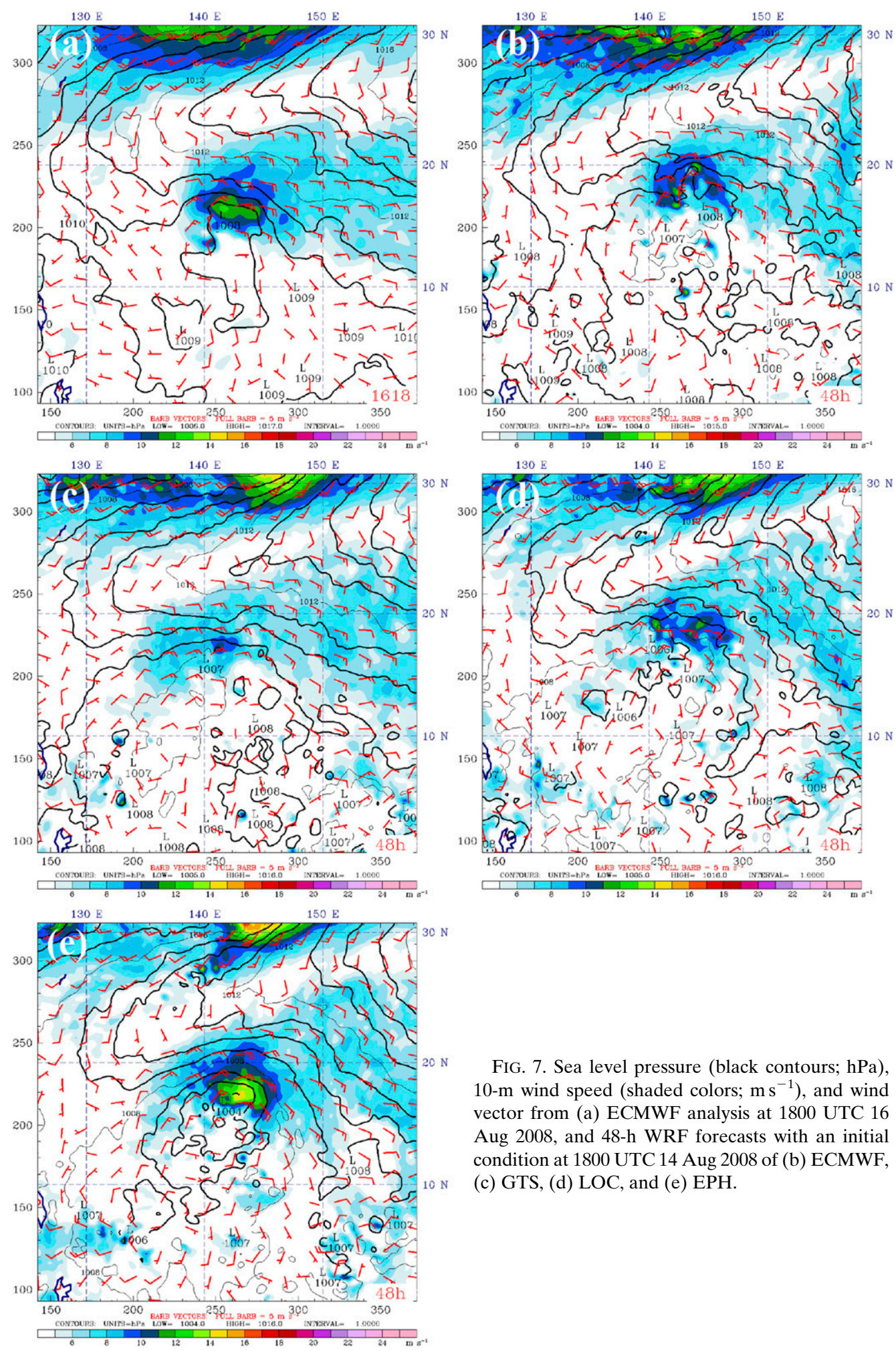

FIG. 7. Sea level pressure (black contours; hPa), $10-\mathrm{m}$ wind speed (shaded colors; $\mathrm{m} \mathrm{s}^{-1}$ ), and wind vector from (a) ECMWF analysis at 1800 UTC 16 Aug 2008, and 48-h WRF forecasts with an initial condition at 1800 UTC 14 Aug 2008 of (b) ECMWF, (c) GTS, (d) LOC, and (e) EPH. 


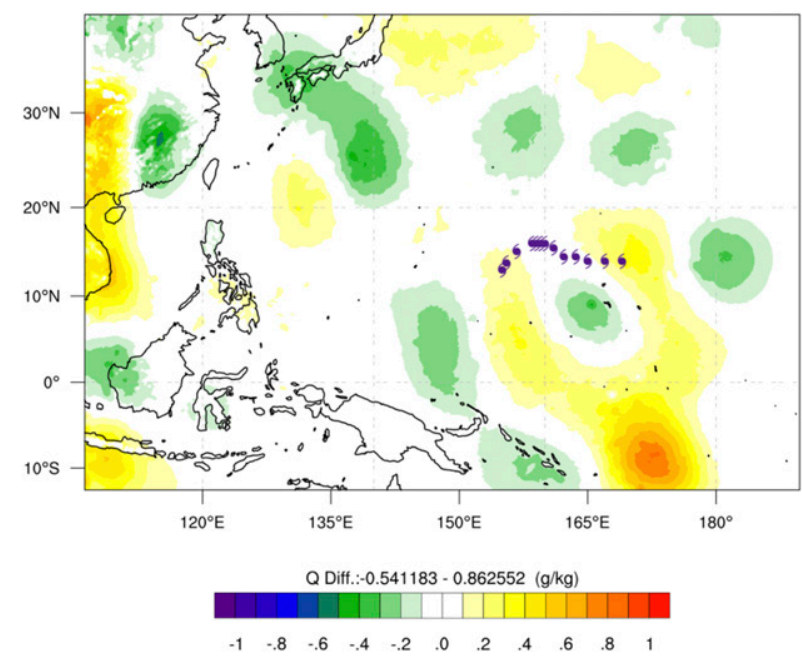

FIG. 8. Time-averaged analysis-increment differences from EPH and LOC in $850-\mathrm{hPa}$ water vapor mixing ratio $\left(\mathrm{g} \mathrm{kg}^{-1}\right)$ during the 3-day data assimilation for Typhoon Nuri. The typhoon symbols are the 500-hPa vortex centers from EPH.

weaker. None of the WRF experiments in this study assimilates satellite radiance, but some of them successfully predict a genesis. This suggests that the RO data could provide complementary information to improve the regional data analysis and prediction.

To gain further insights on the impact of GPS RO operators, we calculate the time-averaged differences in analysis increments for 850 -hPa water vapor mixing ratio between EPH and LOC (Fig. 8), which is defined as

$$
\overline{\sum_{t}\left[\mathrm{INC}(\text { var })_{\mathrm{EPH}}-\mathrm{INC}(\mathrm{var})_{\mathrm{LOC}}\right]},
$$

where var represents a variable and INC is the increment of the variable after assimilation. Correspondingly, Fig. 9 shows the time-averaged differences in the analyses of 850-hPa water vapor mixing ratio (Fig. 9a) and temperature (Fig. 9b) between EPH and LOC. Figure 8 shows that $\mathrm{EPH}$ produces more moisture increment than LOC in the vicinity of the incipient cyclone averaged over the 3-day assimilation period. Similar patterns can be found in Fig. 9a with higher moisture in the EPH analysis along the track of the incipient storm. While the time-averaged difference in the water vapor increment along the storm track is not large, generally less than $0.4 \mathrm{~g} \mathrm{~kg}^{-1}$ (Fig. 8), the resulting time-averaged analysis difference is nearly doubled (Fig. 9a). This reflects the accumulative effect of the analysis increments as well as the contribution of the model physics. In terms of temperature, the timeaveraged analysis difference presents warmer temperature in EPH (Fig. 9b) in the storm environment. The warmer temperature in the lower troposphere allows
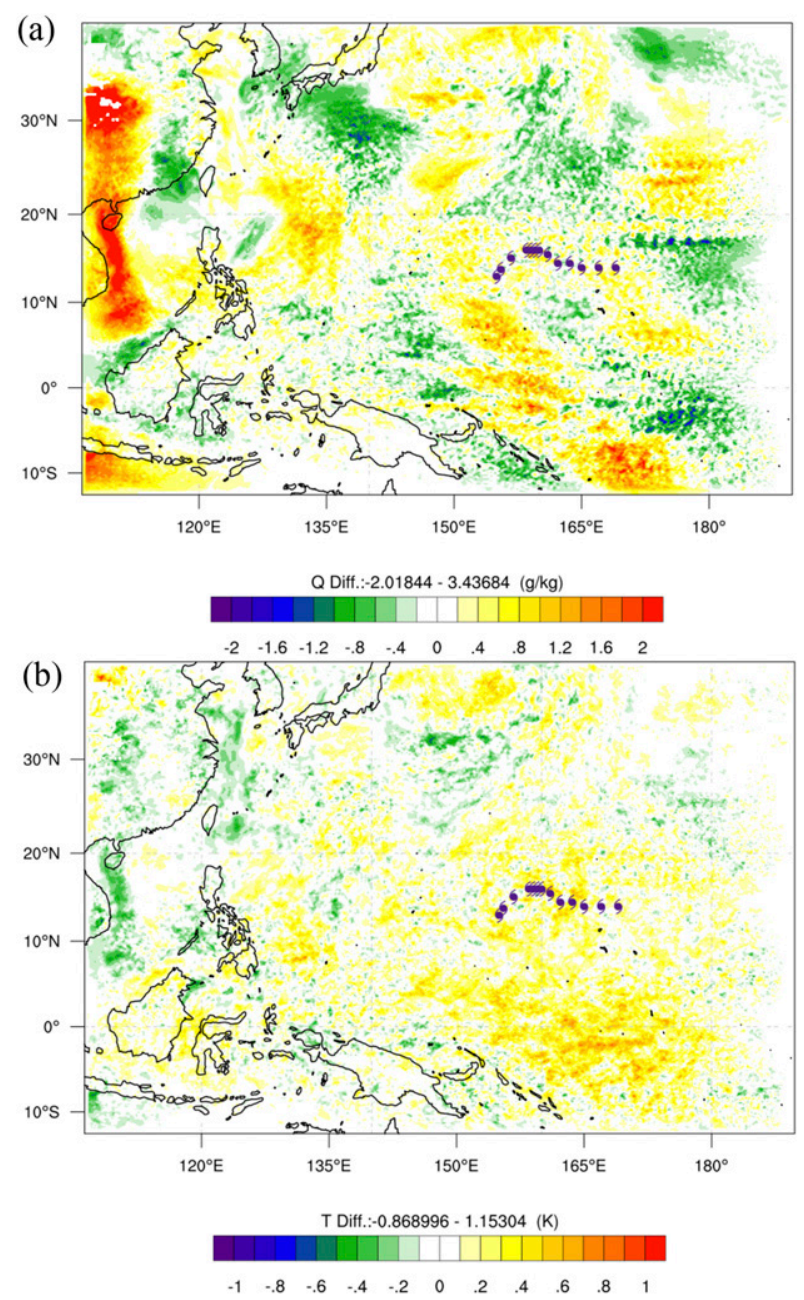

FIG. 9. As in Fig. 8, but for the time-averaged differences in the analyses of $850-\mathrm{hPa}$ (a) water vapor mixing ratio $\left(\mathrm{g} \mathrm{kg}^{-1}\right)$ and (b) temperature $(\mathrm{K})$.

for higher saturation moisture. Consequently, the EPH produces an analysis that is more favorable for tropical cyclogenesis compared with LOC, as shown in Fig. 7. Our results are consistent with $\mathrm{Li}$ and $\mathrm{Pu}$ (2014), which showed that latent heat release associated with moisture is an important factor for the formation of Nuri. We note that the assimilation of GPS RO data using the EPH operator does not always produce more moisture than the LOC operator. The moisture analysis increments can vary depending on the accuracy of the background and the accuracy of the observation operator, that is, different moisture increments in different cases. For example, EPH produces drier time-averaged moisture analysis relative to LOC near the incipient region for Typhoon Fanapi (2010) (figures not shown).

Figure 10 shows the best track for Nuri from JTWC and the simulated track of the $500-\mathrm{hPa}$ vortex from 


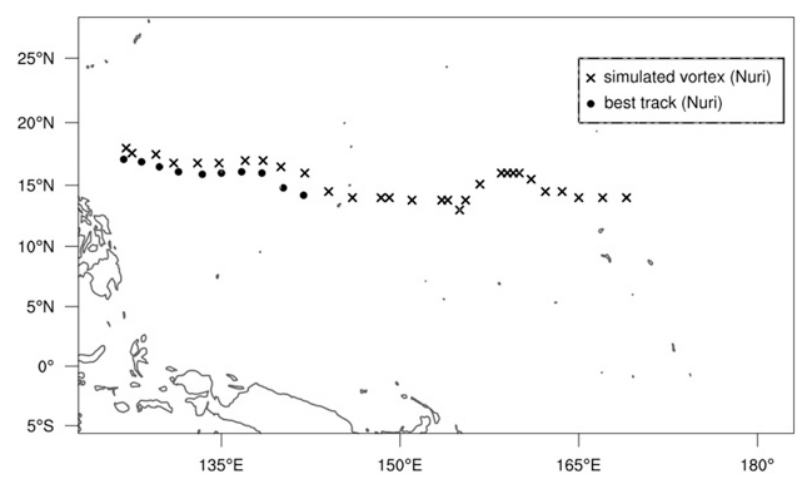

FIG. 10. The best track from JTWC (dots) and simulated track of the 500-hPa vortex from EPH (crosses) for Typhoon Nuri (2008). The JTWC best track starts from 1800 UTC 16 Aug 2008, and the simulated track starts from 1800 UTC 11 Aug 2008.

EPH. Note that the JTWC's best track starts from the time of TD genesis, but the simulated track starts from beginning time of data assimilation. The simulated track is close to the best track, except for the early stage of the TD. In addition, the simulated track of LOC after genesis is close to that of EPH (figure not shown). Figure 11 shows the 6-h accumulated precipitation from grid-scalemicrophysics, subgrid-cumulus, and total precipitation for GTS, LOC, and EPH. It is interesting to note that the amount of subgrid-scale convective precipitation is compatible among GTS, LOC, and EPH. The resolvable-scale precipitation is the differentiator, with GTS the lowest, $\mathrm{EPH}$ the highest, and LOC in the middle. This is true both during the assimilation period and the free forecast period. The assimilation of GPS RO data is able to produce more grid-scale saturation, allowing grid-scale heating to interact positively with the dynamics of the developing vortex. This is apparently very important to the prediction of tropical cyclogenesis, because the genesis was not predicted by GTS and was delayed by $30 \mathrm{~h}$ by LOC.

To further examine the influence of the GPS RO data assimilation, Fig. 12 shows the differences in moisture, vertical velocity, and relative vorticity between $\mathrm{EPH}$ and GTS in a $6^{\circ} \times 6^{\circ}$ region centered on the $500-\mathrm{hPa}$ vortex of EPH (as in Fig. 10). During the 72-h data assimilation, EPH produces an initial condition with higher humidity below $500 \mathrm{hPa}$ (Fig. 12a), and the maximum difference in water vapor mixing ratio between EPH and GTS reaches $2.5 \mathrm{~g} \mathrm{~kg}^{-1}$ at $t=-30 \mathrm{~h}$. Shortly after that, the EPH produces much stronger vertical motion, extending from about 600 to $150 \mathrm{hPa}$, while their difference in moisture below $500 \mathrm{hPa}$ decreases with time. This suggests that the significant increase of moisture in the lower troposphere associated with the assimilation of GPS RO data using the EPH operator is able to enhance organized convection, inducing robust grid-scale
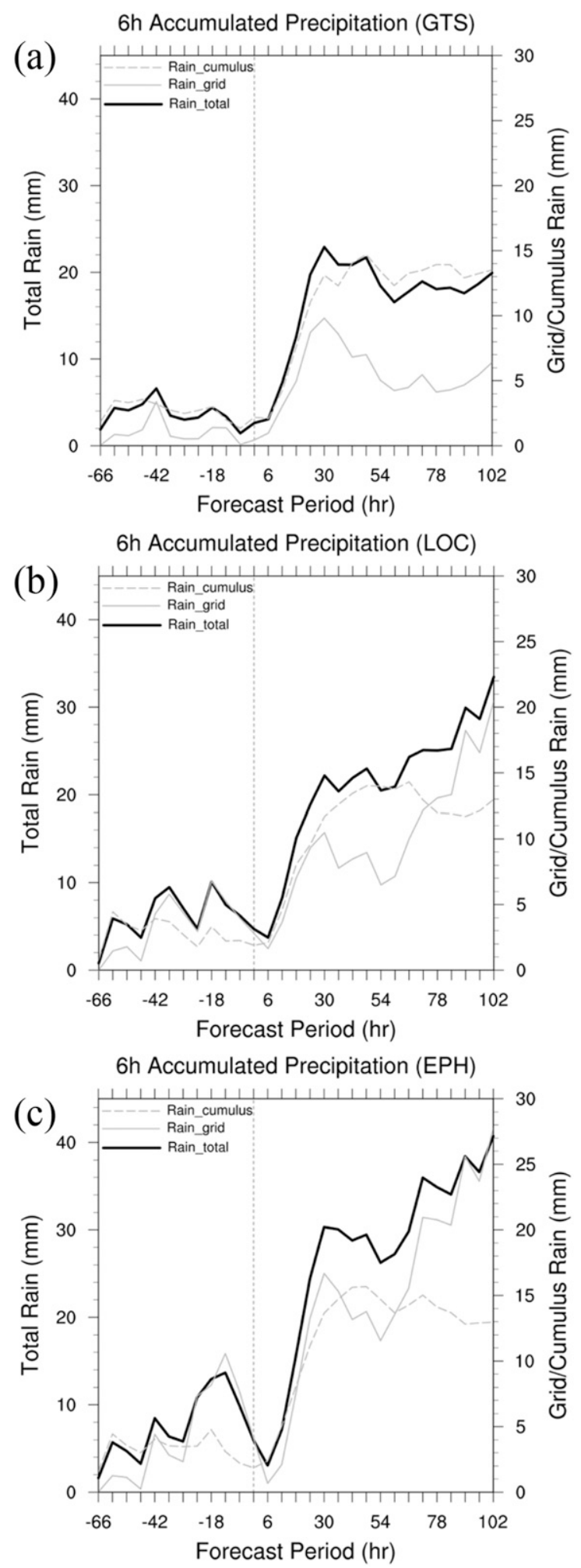

FIG. 11. The 6-h accumulated rainfall from grid-scale, cumulusparameterization, and total precipitation for (a) GTS, (b) LOC, and (c) EPH for Typhoon Nuri. 
(a)

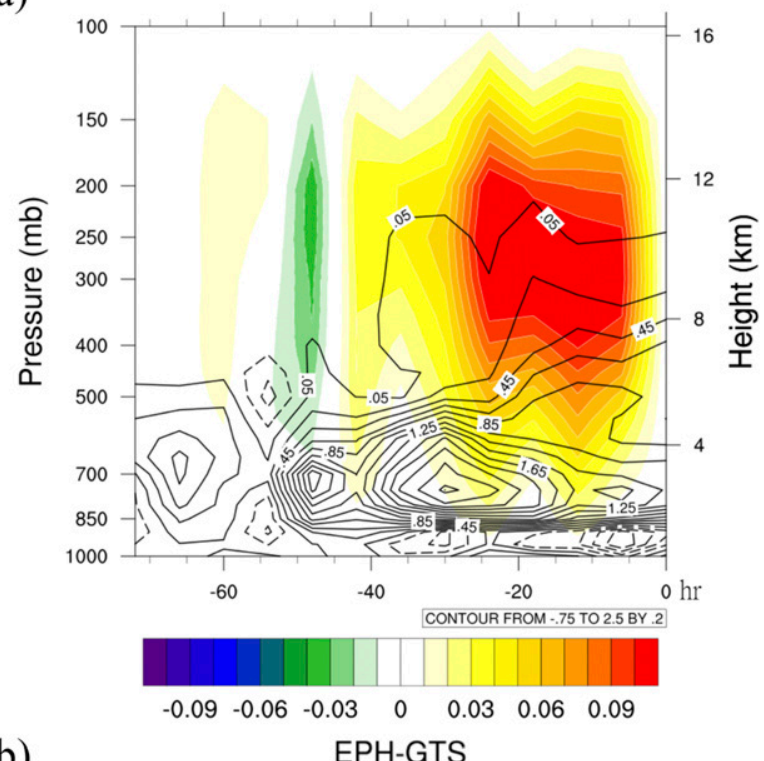

(b)

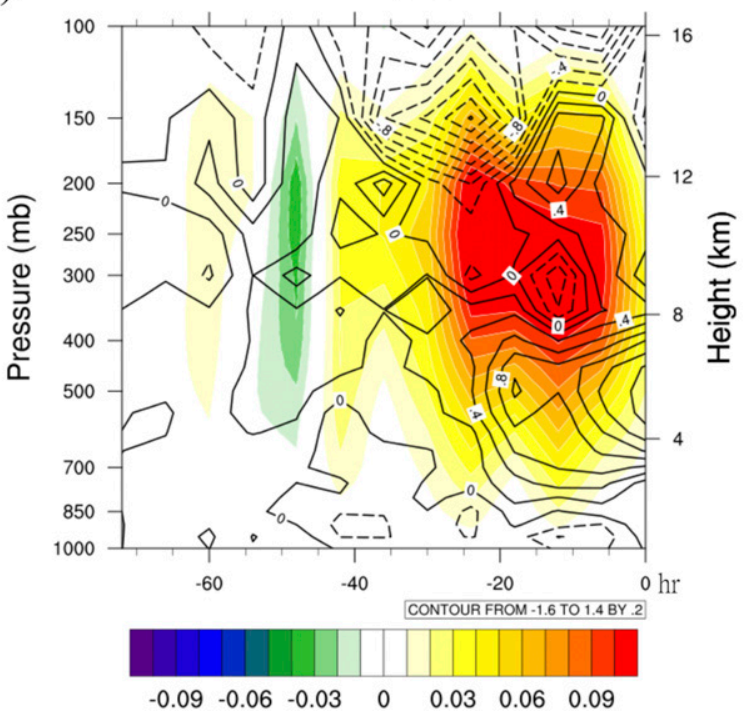

FIG. 12. Differences between the EPH and GTS experiments shown by a time-pressure section during the assimilation period (from -72 to $0 \mathrm{~h}$ ) for Typhoon Nuri (2008) in (a) vertical velocity (colors; $\mathrm{m} \mathrm{s}^{-1}$ ) and water vapor mixing ratio (contours; $\mathrm{g} \mathrm{kg}^{-1}$ ), and (b) vertical velocity (colors; $\mathrm{m} \mathrm{s}^{-1}$ ) and vorticity (contours, $10^{-5} \mathrm{~s}^{-1}$ ). The $0 \mathrm{~h}$ on the $x$ axis indicates the end time of data assimilation cycling and the start time for a free forecast.

vertical motion. The strong vertical motion in turn spins up positive vorticity in the middle troposphere from 700 to $400 \mathrm{hPa}$ during the last day of the assimilation (Fig. 12b). After $72 \mathrm{~h}$ of data assimilation of GPS RO data using the nonlocal observation operator, we have a robust midlevel vortex, coupled with ample moisture in the lower and middle troposphere. The environment is primed to support the tropical

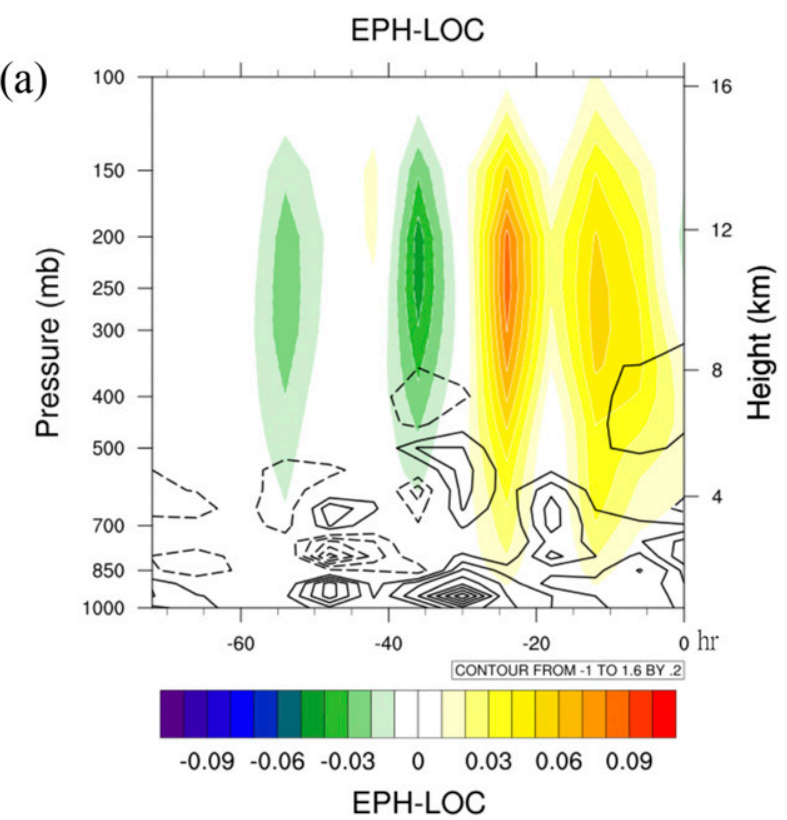

(b)

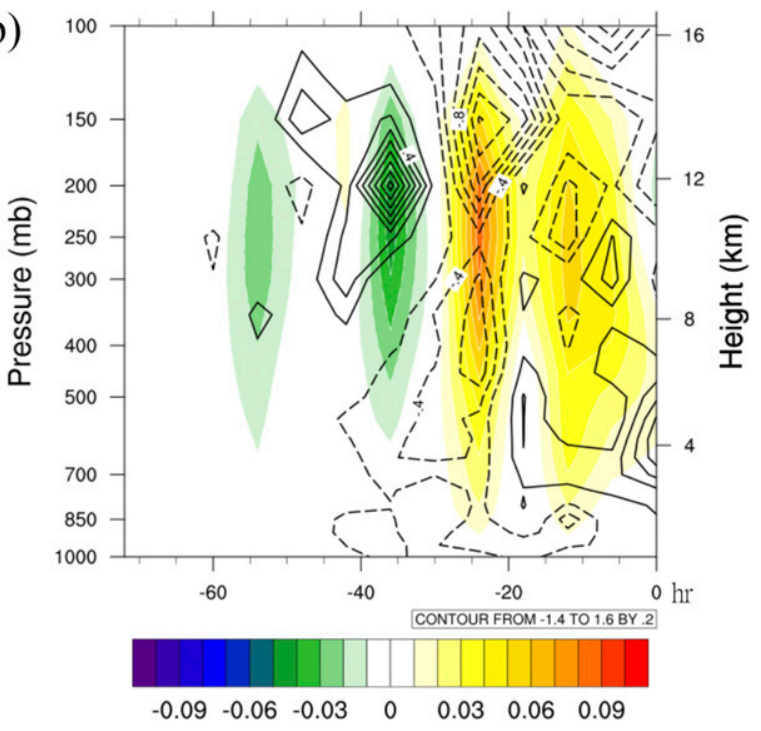

FIG. 13. As in Fig. 12, but for the difference between EPH and LOC.

cyclogenesis. This helps explain why the tropical cyclogenesis is captured in EPH but fails in GTS.

Comparison between LOC and EPH indicates that EPH produces stronger moisture increase (particularly near the ocean surface), stronger vertical motion, and subsequently, stronger midlevel vorticity development (Fig. 13). This provides an explanation of why cyclogenesis predicted by LOC is delayed by $30 \mathrm{~h}$ (Table 1 ). Clearly, assimilation of GPS RO data with the nonlocal excess phase operator is advantageous over the local refractivity operator and is very important for tropical cyclone prediction. 


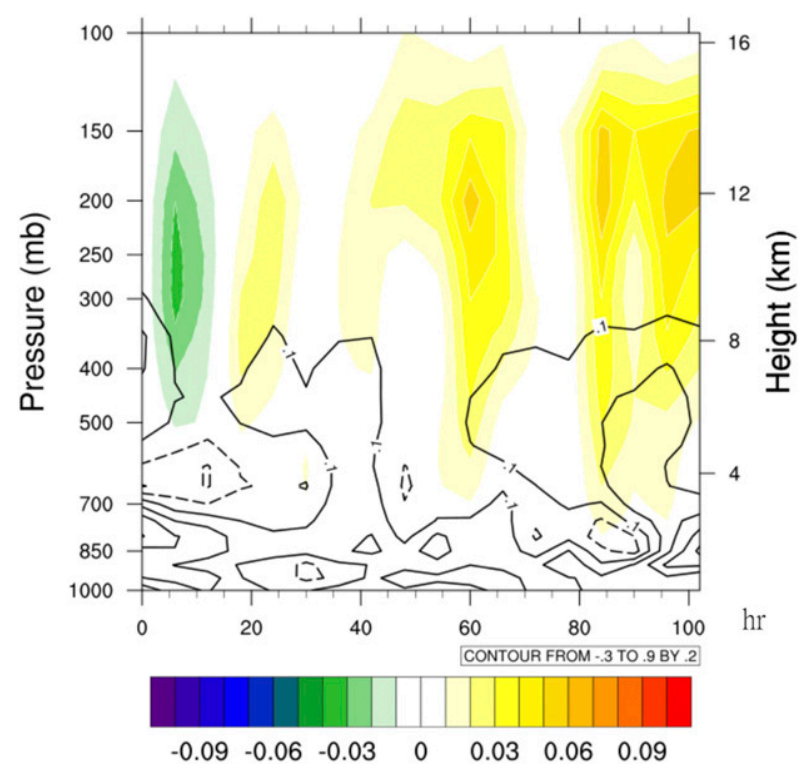

FIG. 14. Differences in vertical velocity (colors; $\mathrm{m} \mathrm{s}^{-1}$ ) and water vapor mixing ratio (contours; $\mathrm{g} \mathrm{kg}^{-1}$ ) between $\mathrm{EPH}$ and EPH.QfromLOC during the forecast period for Typhoon Nuri (2008).

\section{c. Moisture effect}

With the assimilation of GPS RO data, all the variables are modified. The GPS refractivity (and the excess phase) is a function of temperature, pressure, and water vapor; the assimilation of GPS RO data will induce changes in the height field (through the temperature analysis increment). Wind fields, though not directly related to GPS reflectivity, will also be changed through multivariate background error covariances and through geostrophic adjustment (caused by height field changes). From the analysis shown earlier, the assimilation of GPS RO data using EPH operator produces a significant increase of moisture in the lower troposphere, which is important to the development of convection. An interesting question is, Is the improved prediction of cyclogenesis caused primarily by changes in moisture? Or, are the changes of wind and height (i.e., dynamic variables) just as important? To answer these questions, we replace the moisture fields in the final analysis of EPH by that of LOC ("EPH.QfromLOC") while keeping other variables unchanged. The moisture fields include the mixing ratios for water vapor, cloud, rain, snow, and ice. Figure 14 shows that replacing the moisture fields of EPH by those from LOC causes a reduction of lowertroposphere moisture in the subsequent forecast and weaker vertical motion in middle to upper troposphere. Consequently, the genesis is delayed by $18 \mathrm{~h}$ (Table 3 ). In a reversed experiment, replacing the moisture fields of LOC by those of EPH, that is, "LOC.QfromEPH," leads to an improvement in the timing of genesis (from 30-h delay to 24-h delay). Slight supersaturation is presented at a small number of grid points in the initial field for LOC.QfromEPH, but it disappears almost immediately after the model integration.

The results of these moisture-exchange experiments suggest that while the changes of moisture as a result of GPS RO data assimilation is critical, the changes in temperature, height, and wind fields are just as important. Given the fact that LOC.QfromEPH has the same moisture analysis as EPH, the differences lie in temperature, height, and wind fields (i.e., dynamic variables). It implies that the moisture in itself is not sufficient to produce the genesis if the dynamic variables are not accurate.

\section{Summary}

In this study, we assess the impact of RO data assimilation on the formation of 10 tropical cyclones over the northwestern Pacific during 2008-10. Specifically, we conducted three data assimilation experiments. The first experiment only assimilates conventional data from GTS without the use of GPS RO data. The second experiment assimilates GPS RO data using a nonlocal excess phase observation operator $(\mathrm{EPH})$ in addition to the assimilation of the GTS data. The third experiment is similar to that of the second experiment, except a local GPS RO refractivity observation operator is used instead. The results show that the assimilation of GPS RO data using the nonlocal excess phase operator increased the probability of detection of tropical cyclogenesis from $30 \%$ to $70 \%$, while the assimilation of GPS RO data using the local observation operator only increases to $40 \%$. This clearly shows that GPS RO data are quite valuable for the prediction of tropical cyclogenesis.

TABLE 3. Sensitivity tests for Typhoon Nuri (2008). The genesis time with a positive hour indicates a delay relative to the observed, i.e., TD genesis time in JTWC. The observational error percentage is as in Fig. 2.

\begin{tabular}{|c|c|c|c|c|}
\hline Expt & Integral along ray path & Obs error percentage & Other comments & Genesis time \\
\hline LOC.QfromEPH & NO & LOCAL & $\begin{array}{l}\text { Moisture fields exchanged from EPH } \\
\text { at the last cycling run }\end{array}$ & NURI: 24 h \\
\hline EPH.QfromLOC & YES & NONLOCAL & $\begin{array}{l}\text { Moisture fields exchanged from LOC } \\
\text { at the last cycling run }\end{array}$ & NURI: $18 \mathrm{~h}$ \\
\hline
\end{tabular}


Also, the observation operator can have a significant impact on the performance of GPS RO data assimilation.

Among the 10 typhoon cases, Nuri (2008), Sinlaku (2008), and Fanapi (2010) are well captured by EPH but failed by assimilation of GTS only. To assess the importance of GPS RO soundings in the vicinity of the incipient cyclone, we remove GPS RO data within $500 \mathrm{~km}$ of the $500 \mathrm{hPa}$ vortex during the 3-day data assimilation period. Interestingly, all of them fail to form. This illustrates that the data near the incipient cyclone are critical for the prediction of cyclogenesis.

Using Nuri (2008) as an example, we found that the assimilation of GPS RO data using the nonlocal excess phase operator increases the moisture in the lower to middle troposphere substantially. About two days into the assimilation, the increase of moisture induces organized convection, develops robust vertical motion, and causes a significant increase in relative vorticity in the middle troposphere, creating an environment very favorable for tropical cyclogenesis. The assimilation of GPS RO data using the local refractivity operator produces less increase in lower-tropospheric moisture, weaker convection, and weaker vertical motion. As a result, the cyclogenesis is delayed by $30 \mathrm{~h}$.

Additional forecast experiments by exchanging the moisture fields between data assimilation experiments using local and nonlocal observation operators indicate that while moisture is important, the changes in the dynamic variables (i.e., wind, temperature, and height fields) are just as important. The assimilation of GPS RO data using the nonlocal observation operator has produced a strong middle vortex coupled with strong mid- and low-level moisture, which is favorable for tropical cyclogenesis. Decoupling the dynamic variables from the moisture will cause the cyclogenesis to be delayed.

As a cautionary note, this study did not make use of satellite radiance data. The data assimilation experiments are significantly different from that of a modern operational global data assimilation system. As a result, the impact of GPS RO data may be overestimated in the absence of radiance assimilation. Moreover, for a complete assessment of GPS RO data impacts on tropical cyclogenesis, an investigation of the RO data impact on nondeveloping cases should be conducted to see if RO data can reduce false alarms in cyclogenesis prediction. These important issues will be addressed in a future study.

The FORMOSAT-7/COSMIC-2, a follow-on mission of FORMOSAT-3/COSMIC, was launched on 25 June 2019 at an inclination angle of $24^{\circ}$. It is now operational and providing about $5000 \mathrm{RO}$ observations a day over the tropics from six satellites. This offers a great opportunity for the research and operational prediction of tropical cyclones.

Acknowledgments. This research was supported jointly by the Ministry of Science and Technology (MOST) under Grant MOST 107-2111-M-008-017 and National Space Organization (NSPO) under Grant NSPO-S-107049 in Taiwan. It was also supported by the National Science Foundation under Cooperative Agreement 1522830 and the National Space Organization (NSPO) in Taiwan via the American Institute in Taiwan (AIT) under NSPO-UCAR AIT-TECRO Agreement Implementing Arrangement 5.

\section{REFERENCES}

Anderson, J. L., 2010: A non-Gaussian ensemble filter update for data assimilation. Mon. Wea. Rev., 138, 4186-4198, https:// doi.org/10.1175/2010MWR3253.1.

Anthes, R. A., 2011: Exploring Earth's atmosphere with radio occultation: Contributions to weather, climate and space weather. Atmos. Meas. Tech., 4, 1077-1103, https://doi.org/ 10.5194/amt-4-1077-2011.

Aparicio, J. M., and G. Deblonde, 2008: Impact of the assimilation of CHAMP refractivity profiles on Environment Canada global forecasts. Mon. Wea. Rev., 136, 257-275, https://doi.org/ 10.1175/2007MWR1951.1.

Barker, D. M., W. Huang, Y.-R. Guo, A. J. Bourgeois, and Q. N. Xiao, 2004: A three-dimensional variational data assimilation system for MM5: Implementation and initial results. Mon. Wea. Rev., 132, 897-914, https://doi.org/10.1175/1520-0493(2004) 132<0897:ATVDAS > 2.0.CO;2.

, and Coauthors, 2012: The Weather Research and Forecasting Model's Community Variational/Ensemble Data Assimilation System: WRFDA. Bull. Amer. Meteor. Soc., 93, 831-843, https:// doi.org/10.1175/BAMS-D-11-00167.1.

Cardinali, C., 2009: Forecast sensitivity to observation (FSO) as a diagnostic tool. ECMWF Tech. Memo. 599, 28 pp., https:// www.ecmwf.int/node/8574.

, 2013: Observation impact on the short range forecast. ECMWF Lecture Notes, 17 pp., https://www.ecmwf.int/node/ 16937.

Chen, F., and J. Dudhia, 2001: Coupling an advanced land surfacehydrology model with the Penn State-NCAR MM5 modeling system. Part I: Model implementation and sensitivity. Mon. Wea. Rev., 129, 569-585, https://doi.org/10.1175/15200493(2001)129<0569:CAALSH > 2.0.CO;2.

Chen, S.-Y., C.-Y. Huang, Y.-H. Kuo, Y.-R. Guo, and S. Sokolovskiy, 2009: Assimilation of GPS refractivity from FORMOSAT-3/COSMIC using a nonlocal operator with WRF 3DVAR and its impact on the prediction of a typhoon event. Terr. Atmos. Oceanic Sci., 20, 133-154, https://doi.org/ 10.3319/TAO.2007.11.29.01(F3C).

$-, \ldots,-$, and S. Sokolovskiy, 2011: Observational error estimation of FORMOSAT-3/COSMIC GPS radio occultation data. Mon. Wea. Rev., 139, 853-865, https://doi.org/ 10.1175/2010MWR3260.1.

- T.-K. Wee, Y.-H. Kuo, and D. H. Bromwich, 2014: An impact assessment of GPS radio occultation data on prediction of a rapidly developing cyclone over the Southern Ocean. Mon. Wea. Rev., 142, 4187-4206, https://doi.org/10.1175/MWR-D14-00024.1. 
Chen, Y.-C., M.-E. Hsieh, L.-F. Hsiao, Y.-H. Kuo, M.-J. Yang, C.-Y. Huang, and C.-S. Lee, 2015: Systematic evaluation of the impacts of GPSRO data on the prediction of typhoons over the northwestern Pacific in 2008-2010. Atmos. Meas. Tech., 8, 2531-2542, https://doi.org/10.5194/amt-8-2531-2015.

Chou, M.-D., and M. J. Suarez, 1994: An efficient thermal infrared radiation parameterization for use in general circulation models. Series on Global Modeling and Data Assimilation, Vol. 3, NASA Tech. Memo. 104606, 85 pp., https:/ntrs.nasa.gov/archive/nasa/ casi.ntrs.nasa.gov/19950009331.pdf.

COSMIC, 2013: Atmospheric profilè from COSMIC occultation data, continuing from April 2006 (updated daily). COSMIC Climate Data Analysis and Archive Center, accessed 5 August 2014, http://rda.ucar.edu/datasets/ds723.0/.

Cucurull, L., J. C. Derber, R. Treadon, and R. J. Purser, 2007: Assimilation of global positioning system radio occultation observations into NCEP's Global Data Assimilation System. Mon. Wea. Rev., 135, 3174-3193, https://doi.org/10.1175/ MWR3461.1.

- — - and R. J. Purser, 2013: A bending angle forward operator for global positioning system radio occultation measurements. J. Geophys. Res. Atmos., 118, 14-28, https:// doi.org/10.1029/2012JD017782.

—, R. A. Anthes, and L.-L. Tsao, 2014: Radio occultation observations as anchor observations in numerical weather prediction models and associated reduction of bias corrections in microwave and infrared satellite observations. J. Atmos. Oceanic Technol., 31, 20-32, https://doi.org/10.1175/JTECHD-13-00059.1.

Doyle, J. D., C. A. Reynolds, C. Amerault, and J. Moskaitis, 2012: Adjoint sensitivity and predictability of tropical cyclogenesis. J. Atmos. Sci., 69, 3535-3557, https://doi.org/10.1175/JAS-D12-0110.1.

Halperin, D. J., H. E. Fuelberg, R. E. Hart, J. H. Cossuth, P. Sura, and R. J. Pasch, 2013: An evaluation of tropical cyclone genesis forecasts from global numerical models. Wea. Forecasting, 28, 1423-1445, https://doi.org/10.1175/WAF-D-13-00008.1.

,,$-- \ldots$, and,- 2016 : Verification of tropical cyclone genesis forecasts from global numerical models: Comparisons between the North Atlantic and eastern North Pacific basins. Wea. Forecasting, 31, 947-955, https://doi.org/10.1175/WAFD-15-0157.1.

Healy, S. B., 2013: Surface pressure information retrieved from GPS radio occultation measurements. Quart. J. Roy. Meteor. Soc., 139, 2108-2118, https://doi.org/10.1002/qj.2090.

— CHAMP GPS radio occultation measurements. Quart. J. Roy. Meteor. Soc., 132, 605-623, https://doi.org/10.1256/qj.04.182.

_ J. R. Eyre, M. Hamrud, and J. N. Thépaut, 2007: Assimilating GPS radio occultation measurements with two-dimensional bending angle observation operators. Quart. J. Roy. Meteor. Soc., 133, 1213-1227, https://doi.org/10.1002/qj.63.

Hong, S.-Y., Y. Noh, and J. Dudhia, 2006: A new vertical diffusion package with an explicit treatment of entrainment processes. Mon. Wea. Rev., 134, 2318-2341, https://doi.org/10.1175/ MWR3199.1.

Huang, C.-Y., Y.-H. Kuo, S.-H. Chen, and F. Vandenberghe, 2005: Improvements in typhoon forecasts with assimilated GPS occultation refractivity. Wea. Forecasting, 20, 931-953, https:// doi.org/10.1175/WAF874.1.

, and Coauthors, 2010: Impact of GPS radio occultation data assimilation on regional weather predictions. GPS Solutions, 14, 35-49, https://doi.org/10.1007/s10291-009-0144-1.
— S.-Y. Chen, S. K. A. V. Prasad Rao Anisetty, S.-C. Yang, and L.-F. Hsiao, 2016: An impact study of GPS radio occultation observations on frontal rainfall prediction with a local bending angle operator. Wea. Forecasting, 31, 129-150, https://doi.org/ 10.1175/WAF-D-15-0085.1.

Huang, X.-Y., and Coauthors, 2009: Four-dimensional variational data assimilation for WRF: Formulation and preliminary results. Mon. Wea. Rev., 137, 299-314, https://doi.org/10.1175/ 2008MWR2577.1.

Kain, J. S., and J. M. Fritsch, 1990: A one-dimensional entraining/ detraining plume model and its application in convective parameterization. J. Atmos. Sci., 47, 2784-2802, https://doi.org/ 10.1175/1520-0469(1990)047<2784:AODEPM>2.0.CO;2.

— , and _ 1993: Convective parameterization for mesoscale models: The Kain-Fritsch scheme. The Representation of Cumulus Convection in Numerical Models, Meteor. Monogr., No. 16, Amer. Meteor. Soc., 165-170, https://doi.org/10.1007/ 978-1-935704-13-3_16.

Kueh, M.-T., C.-Y. Huang, S.-Y. Chen, S.-H. Chen, and C.-J. Wang, 2009: Impact of GPS radio occultation refractivity soundings on a simulation of Typhoon Bilis (2006) upon landfall. Terr. Atmos. Oceanic Sci., 20, 115-131, https://doi.org/ 10.3319/TAO.2008.01.21.03(F3C).

Kuo, Y. H., T. K. Wee, S. Sokolovskiy, C. Rocken, W. Schreiner, D. Hunt, and R. A. Anthes, 2004: Inversion and error estimation of GPS radio occultation data. J. Meteor. Soc. Japan, 82, 507-531, https://doi.org/10.2151/jmsj.2004.507.

Kursinski, E. R., G. A. Hajj, J. T. Schofield, R. P. Linfield, and K. R. Hardy, 1997: Observing Earth's atmosphere with radio occultation measurements using the global positioning system. J. Geophys. Res., 102, 23 429-23 465, https://doi.org/10.1029/ 97JD01569.

Lewis, H., 2008: Refractivity calculations in ROPP. GRAS SAF Rep. 05, 8 pp., http://www.romsaf.org/general-documents/gsr/ gsr_05.pdf.

Li, Z., and Z. Pu, 2014: Numerical simulations of the genesis of Typhoon Nuri (2008): Sensitivity to initial conditions and implications for the roles of intense convection and moisture conditions. Wea. Forecasting, 29, 1402-1424, https://doi.org/ 10.1175/WAF-D-14-00003.1.

Lin, Y.-L., R. D. Farley, and H. D. Orville, 1983: Bulk parameterization of the snow field in a cloud model. J. Climate Appl. Meteor., 22, 1065-1092, https://doi.org/10.1175/15200450(1983)022<1065:BPOTSF $>2.0$.CO;2.

Liu, H., J. Anderson, and Y.-H. Kuo, 2012: Improved analyses and forecasts of Hurricane Ernesto's genesis using radio occultation data in an ensemble filter assimilation system. Mon. Wea. Rev., 140, 151-166, https://doi.org/10.1175/MWRD-11-00024.1.

Lussier, L. L., III, M. T. Montgomery, and M. M. Bell, 2014: The genesis of Typhoon Nuri as observed during the Tropical Cyclone Structure 2008 (TCS-08) field experiment. Part III: Dynamics of low-level spin-up during the genesis. Atmos. Chem. Phys., 14, 8795-8812, https://doi.org/10.5194/acp-14-8795-2014.

Mlawer, E. J., S. J. Taubman, P. D. Brown, M. J. Iacono, and S. A. Clough, 1997: Radiative transfer for inhomogeneous atmospheres: RRTM, a validated correlated- $k$ model for the longwave. J. Geophys. Res., 102, 16 663-16 682, https://doi.org/ 10.1029/97JD00237.

Poli, P., S. B. Healy, F. Rabier, and J. Pailleux, 2008: Preliminary assessment of the scalability of GPS radio occultations impact in numerical weather prediction. Geophys. Res. Lett., 35, L23811, https://doi.org/10.1029/2008GL035873. 
Raymond, D. J., and C. López Carrillo, 2011: Vorticity budget of Typhoon Nuri. Atmos. Chem. Phys., 11, 147-163, https:// doi.org/10.5194/acp-11-147-2011.

Rennie, M. P., 2010: The impact of GPS radio occultation assimilation at the Met Office. Quart. J. Roy. Meteor. Soc., 136, 116-131, https://doi.org/10.1002/qj.521.

Rutledge, S. A., and P. V. Hobbs, 1984: The mesoscale and microscale structure and organization of clouds and precipitation in midlatitude cyclones. XII: A diagnostic modeling study of precipitation development in narrow cold-frontal rainbands. J. Atmos. Sci., 41, 2949-2972, https://doi.org/10.1175/15200469(1984)041<2949:TMAMSA > 2.0.CO;2.

Sippel, J. A., and F. Zhang, 2008: A probabilistic analysis of the dynamics and predictability of typhoon cyclogenesis. J. Atmos. Sci., 65, 3440-3459, https://doi.org/10.1175/2008JAS2597.1.

Skamarock, W. C., and Coauthors, 2008: A description of the Advanced Research WRF version 3. NCAR Tech. Note NCAR/TN-475+STR, 113 pp., https://doi.org/10.5065/D68S4MVH.

Sokolovskiy, S., Y.-H. Kuo, and W. Wang, 2005a: Assessing the accuracy of a linearized observation operator for assimilation of radio occultation data: Case simulations with a highresolution weather model. Mon. Wea. Rev., 133, 2200-2212, https://doi.org/10.1175/MWR2948.1.

,$- \ldots$, and,$- 2005 \mathrm{~b}$ : Evaluation of a linear phase observation operator with CHAMP radio occultation data and highresolution regional analysis. Mon. Wea. Rev., 133, 3053-3059, https://doi.org/10.1175/MWR3006.1.

Steiner, A. K., B. C. Lackner, F. Ladstädter, B. Scherllin-Pirscher, U. Foelsche, and G. Kirchengast, 2011: GPS radio occultation for climate monitoring and change detection. Radio Sci., 46, RS0D24, https://doi.org/10.1029/2010RS004614.
Syndergaard, S., Y.-H. Kuo, and M. S. Lohmann, 2006: Observation operators for the assimilation of occultation data into atmospheric models: A review. Atmosphere and Climate, U. Foelsche, G. Kirchengast, and A. K. Steiner, Eds., Springer, 205-224, https://doi.org/10.1007/3-540-34121-8_18.

Tsai, H.-C., K.-C. Lu, R. L. Elsberry, M.-M. Lu, and C.-H. Sui, 2011: Tropical cyclone-like vortices detection in the NCEP 16-day ensemble system over the western North Pacific in 2008: Application and forecast evaluation. Wea. Forecasting, 26, 77-93, https://doi.org/10.1175/2010WAF2222415.1.

Wang, X., D. M. Barker, C. Snyder, and T. M. Hamill, 2008a: A hybrid ETKF-3DVAR data assimilation scheme for the WRF Model. Part I: Observing system simulation experiment. Mon. Wea. Rev., 136, 5116-5131, https://doi.org/ 10.1175/2008MWR2444.1.

,,,--- and,- 2008 b: A hybrid ETKF-3DVAR data assimilation scheme for the WRF Model. Part II: Real observation experiments. Mon. Wea. Rev., 136, 5132-5147, https://doi.org/10.1175/2008MWR2445.1.

Wee, T.-K., Y.-H. Kuo, and D.-K. Lee, 2010: Development of a curved ray tracing method for modeling of phase paths from GPS radio occultation: A two-dimensional study. J. Geophys. Res., 115, D24119, https://doi.org/10.1029/2010JD014419.

Yang, S.-C., S.-H. Chen, S.-Y. Chen, C.-Y. Huang, and C.-S. Chen, 2014: Evaluating the impact of the COSMIC RO bending angle data on predicting the heavy precipitation episode on 16 June 2008 during SoWMEX-IOP8. Mon. Wea. Rev., 142, 4139-4163, https://doi.org/10.1175/MWR-D-13-00275.1.

Zhang, F., and J. A. Sippel, 2009: Effects of moist convection on hurricane predictability. J. Atmos. Sci., 66, 1944-1961, https:// doi.org/10.1175/2009JAS2824.1. 